\title{
I-Optimal Axial Designs for Four Ingredient Concrete Experiment
}

\author{
Njoroge Elizabeth Wambui ${ }^{1,}$, Koske Joseph ${ }^{2}$, Mutiso John ${ }^{2}$ \\ ${ }^{1}$ Department of Physical Sciences, Chuka University, Chuka, Kenya \\ ${ }^{2}$ Department of Mathematics, Physics and Computing, Moi University, Eldoret, Kenya
}

Email address:

elizawn@gmail.com (N. E. Wambui), koske4@yahoo.co.uk (K. Joseph), johnkasome@yahoo.com (M. John)

${ }^{*}$ Corresponding author

\section{To cite this article:}

Njoroge Elizabeth Wambui, Koske Joseph, Mutiso John. I-Optimal Axial Designs for Four Ingredient Concrete Experiment. American Journal of Theoretical and Applied Statistics. Vol. 10, No. 1, 2021, pp. 32-37. doi: 10.11648/j.ajtas.20211001.15

Received: November 13, 2020; Accepted: December 1, 2020; Published: January 28, 2021

\begin{abstract}
Stakeholders in the construction industry work towards obtaining optimal concrete mixes with an aim of producing structures with the best compressive strength. In many instances, Kenya has witnessed collapse of buildings leading to death and huge financial loses, which has been associated largely to poor concrete mixes. This paper aims at evaluating the I-optimal designs for a concrete mixture experiment for both Equally Weighted Simplex Centroid Axial Design and Unequally Weighted Simplex Centroid Axial Design, based on the second-degree Kronecker model. Optimality tests are performed to locate the optimum values of a design. In various studies, I-optimality has been shown to be among the best criteria in obtaining the most optimal outcomes. In this study, Response Surface Methodology is applied in evaluating I-optimal designs, which are known to minimize average or integrated prediction variance over the experimental region. I-optimality equivalence conditions for the inscribed tetrahedral design and for the concrete experiment model are identical with the boundary points, mid-face points and the centroid, denoted by $\eta_{2}, \eta_{3}$ and $\eta_{4}$ respectively. Equally, Weighted Simplex Centroid Axial Design proved to be a more I-efficient design than the Unequally Weighted Simplex Centroid Axial Design for both the tetrahedral design and the concrete model, with $87.85 \%$ and $79.54 \%$ respectively. The optimal response surface occurred in the region of the I-optimal designs. The Kronecker model derived from the concrete mixture experiment proved effective and efficient in describing the observed results.
\end{abstract}

Keywords: I-Optimality, Tetrahedral, Efficiency, Equivalence, Average Prediction

\section{Introduction}

In the general mixture problem, the measured response is assumed to depend only on the proportions of the ingredients present in the mixture and not on the amount of the mixture according to [2]. The mixture ingredients $t_{i}, i=1,2, \ldots, m$ are such that $t_{i} \geq 0$. The experimental region is given by the probability

simplex $T_{m}=\left\{t=\left(t_{1}, \ldots, t_{m}\right)^{\prime} \in[0,1]^{m}: \sum_{i=1}^{m} t_{i}=1\right\}, t \in$ $T_{m}$.

The objectives of the analysis of mixture data are to fit a proposed model for describing the shape of the response surface over the simplex factor space, and to determine the roles played by the individual components also alluded is that the same analysis may achieve these two objectives at once as said by [2]. Axial designs are defined as the designs with interior points $x_{i}=0, x_{j}=\frac{1}{1-q}, \forall j \neq i$ and $x_{i}=1, x_{j}=$ $0, \forall j \neq i$, which contains the points of the form $\left[\frac{1+(q-1) \Delta}{q}, \frac{1-\Delta}{q}, \ldots, \frac{1-\Delta}{q}\right]$ and its permutations $\frac{-1}{q-1}<\Delta<1$, as described by [13].

An optimality criterion is one, which summarizes how good a design is, and it is maximized or minimized by an optimal design. I-optimal criterion is an information-based criterion, and unlike the D-optimal designs the I-optimal designs are not frequently used, as was noted by [12]. The D-optimal designs aims at precise model estimation while the I-optimal designs aims at obtaining precise predictions. For mixture experiments, the focus is to find certain responses for any given components proportions formulations, with an aim of obtaining the optimal responses from optimal settings with the best precision. Dand G-optimal designs for four ingredient mixture, were evaluated by [9]. This paper evaluates the same D-and 
G-optimal designs with I-optimality criteria for a concrete mixture experiment to obtain precise predictions on outcomes.

\section{Methodology}

Some study that applied the Kiefer's functions as optimality criteria to evaluate the designs in third degree Kronecker model mixture experiment for non-maximal subsystem of parameters was by [7]. This study chose I-optimality also known as the Q-optimality and as IV-, V-optimality as called by $[5,3]$ respectively. This optimality had started gaining popularity with experimenters because of its prediction capability as was noted by [6]. An inscribed tetrahedron weighted simplex centroid design suggested by [10] was applied.

The second-degree Kronecker model and its subsystem of interest are given by (1) and (2) respectively. The latter is written in Lexicographic order as suggested by [4]. The model was utilized in obtaining the concrete regression model (18) which was analyzed in this study.

$$
\begin{gathered}
E\left(Y_{t}\right)=f(t)^{\prime} \theta=(t \otimes t)^{\prime} \theta=\sum_{i=1}^{m} \theta_{i i} t_{i}^{2}+\sum_{i, j=1, i<j}^{m}\left(\theta_{i j}+\theta_{j i}\right) t_{i} t_{j} \\
E(Y)=\theta_{11} t_{1}^{2}+\theta_{22} t_{2}^{2}+\theta_{33} t_{3}^{2}+\theta_{44} t_{4}^{2}+\theta_{12} t_{1} t_{2}+\theta_{13} t_{1} t_{3}+\theta_{14} t_{1} t_{4}+\theta_{23} t_{2} t_{3}+\theta_{24} t_{2} t_{4}+\theta_{34} t_{3} t_{4}
\end{gathered}
$$

Two weighted designs for four components namely; Equally Weighted Simplex Centroid Axial Design (EWSCAD) and Unequally Weighted Simplex Centroid Axial Design (UWSCAD) were used to compare the I-optimality conditions for the concrete experiment model, which were evaluated against the I-optimality conditions of the inscribed tetrahedral design.

Direct search for optimum designs could be difficult because it depends on the nature of response function, criterion function and the experimental region as was explained by [14].

The I-optimal criteria develops designs that minimizes the average or integrated prediction variance over the experimental regions given in (3) as given by [1].

$$
\begin{gathered}
\text { Average variance }=\frac{\int_{\chi} f^{T}(x) M(\xi)^{-1} f(x)}{\int_{\chi} d x} \\
B=\int_{s_{q-1}} X_{1}^{p 1}, X_{2}^{p 2}, \ldots, X_{q}^{p q} \\
L_{i j}=K\left(\frac{\prod_{i=1}^{q} p_{i} !}{\left(\sum_{i=1}^{q} p_{i}+q-1\right) !}\right)
\end{gathered}
$$$$
B=\int_{s_{q-1}} X_{1}^{p 1}, X_{2}^{p 2}, \ldots, X_{q}^{p q} d x_{1}, d x_{2}, \ldots, d x p=\frac{\prod_{i=1}^{q} \Gamma\left(p_{i}+1\right)}{\Gamma\left(q+\sum_{i=1}^{q} p_{i}\right)}=\frac{\prod_{i=1}^{q} p_{i} !}{\left(\sum_{i=1}^{q} p_{i}+q-1\right) !}
$$

Average variance $=\operatorname{tr}\left[M^{-1} L\right]$ and $L=\Gamma(q) B . \quad \mathrm{L}$ is the moment matrix since the elements are moments of a uniform distribution on the experimental region $S_{q-1}$, and $\mathrm{M}$ is the information matrix of the full model. $\mathrm{B}$ is the matrix given as

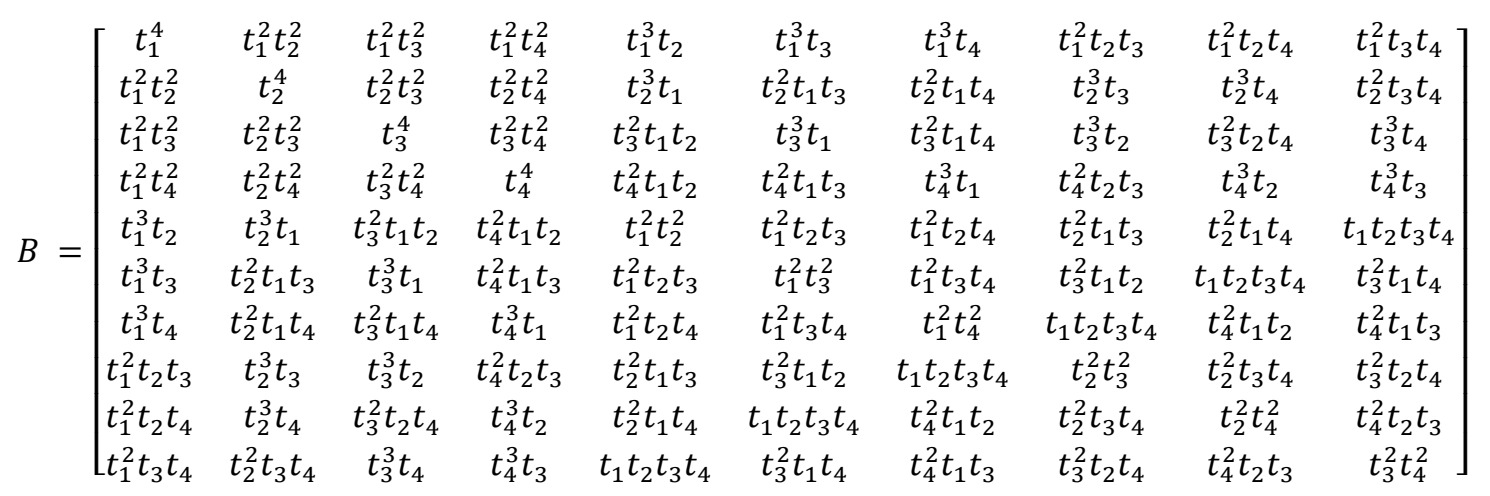

(9). $\Gamma(q)=K=(q-1) !$ and $M=X^{\prime} \Lambda X$, where $X=$ $\left[f\left(t_{1}\right), \ldots, f\left(t_{p}\right)\right]$ is the $p \times p$ model matrix corresponding to $p$ points of the simplex centroid design. $\Lambda=$ $\operatorname{diag}\left(r_{1} I_{11}, \ldots, r_{q} I_{q q}\right), r_{1}, \ldots, r_{q}$ are the weights of the different design points.

$\mathrm{M}$ is the information matrix under the I-Optimality, for the full Kronecker model whose average variance is given by
Since the information matrix $M(\xi)$ in (3) is constant as far as the integration is concerned, the formula for the average variance can also be expressed as (4).

Prediction variance can be rewritten as (5) as was stated by

$$
\text { Ave. } v=\frac{1}{\int_{\chi} d x} \operatorname{trace}\left[M(\xi)^{-1} \int_{\chi} f(x) f^{T}(x) d x\right]
$$

A more convenient way of finding the average variance was obtained as given in (6) as shown by [11].

$$
\text { Average variance }=\frac{1}{\int_{\chi} d x} \operatorname{trace}\left[M(\xi)^{-1} B\right]
$$

The matrix B was obtained by the integral 
The focus of this study was the subsystem of interest, hence the average variance was obtained by

$$
\text { Ave. } \text { variance }=\operatorname{tr}\left[C^{-1} L\right]
$$

$\mathrm{C}$ is the information matrix of the subsystem of interest; this was represented by equations (18) and (19) for EWSCAD and UWSCAD respectively in [9].

The general equivalence theorem provides a methodology to check the optimality of a given continuous design, for any convex and differentiable design optimality criteria.

A continuous design with information matrix $M$ for the full model is I-optimal if and only if

$$
f^{\prime}(t) M^{-1} L M^{-1} f(t) \leq \operatorname{tr}\left(M^{-1} L\right)
$$

As indicated by [1].

The subsystem of interest design points in the experimental region are I-optimal if and only if

$$
f^{\prime}(t) C^{-1} L C^{-1} f(t) \leq \operatorname{tr}\left(C^{-1} L\right)
$$

Efficiency of designs enables to find the better performing designs. Comparing the average variances of prediction $P_{1}$ and $P_{2}$ for the designs $\xi$ and $\xi^{*}$ respectively, the I-efficiency is computed as $I_{\text {eff }}=\frac{P_{2}}{P_{1}} \times 100$. Equation (14) gave the working of I-efficiencies for this study.

$$
I_{e f f}(\tau)=\frac{\operatorname{tr}\left[C^{*-1} L^{*}\left(\xi^{*}\right)\right]}{\operatorname{tr}\left[C^{-1} L(\xi)\right]} \times 100
$$

I-efficiency larger than $100 \%$ indicates that $\xi^{*}$ is better than $\xi$ in terms of the average prediction variance.

A study that obtained the values of the desired optimal design with respect to their corresponding information matrices, discovered that the D-A-E-G optimality designs at the boundary points were better than the designs obtained from interior support points as expressed by [15].

\section{Results and Discussions}

The integrals of the $10 \times 10$ matrix are as shown in set of equations (15). The components are represented by $\mathrm{i}, \mathrm{j}, \mathrm{k}, \mathrm{w}=$ $1,2,3,4$, while $n$ and $m$ represent the rows and columns of the moment matrix $\mathrm{L}$, the constant $\mathrm{K}=6$. The matrix $\mathrm{L}$ is a symmetric matrix along the main diagonal.

$$
\begin{gathered}
M_{n n}=K \int t_{i}^{4} d t_{i}=\frac{3 ! 4 !}{(4+4-1) !}=\frac{1}{35}, i=n=1,2,3,4 \\
M_{n m}=K \int t_{i}^{2} t_{j}^{2} d t_{i} d t_{j}=\frac{1}{210},(i \neq j),(n \neq m)=1,2,3,4 \\
M_{n n}=K \int t_{i}^{2} t_{j}^{2} d t_{i} d t_{j}=\frac{1}{210},(i \neq j), n=5,6,7,8,9,10 \\
M_{n m}=K \int t_{i}^{3} t_{j} d t_{i} d t_{j}=\frac{1}{140}, i \neq j \\
(n, m)=\{(1,5),(1,6),(1,7),(2,5),(2,8),(2,9),(3,6) \\
(3,8),(3,10),(4,7),(4,9),(4,10)\}
\end{gathered}
$$

$$
\begin{gathered}
M_{n m}=K \int t_{i}^{2} t_{j} t_{k} d t_{i} d t_{j} d t_{k}=\frac{1}{420}, i \neq j \neq k \\
(n, m)=\{(1,8),(1,9),(1,10),(2,6),(2,7),(2,10),(3,5), \\
(3,7),(3,9),(4,5),(4,6),(4,8),(5,6),(5,7),(5,8),(5,9), \\
(6,7),(6,8),(6,10),(7,9),(7,10),(8,9),(8,10),(9,10) .
\end{gathered}
$$$$
M_{n m}=K \int t_{i} t_{j} t_{k} t_{w} d t_{i} d t_{j} d t_{k} d t_{w}=\frac{1}{840} .
$$$$
i \neq j \neq k \neq w, \mathrm{w}=4(n, m)=(5,10),(6,9),(7,8), n \neq m \text {. }
$$

The summarized workings in (15) are as shown in the moment matrix (16).

$$
B=\frac{1}{840}\left[\begin{array}{cccccccccc}
24 & 4 & 4 & 4 & 6 & 6 & 6 & 2 & 2 & 2 \\
4 & 24 & 4 & 4 & 6 & 2 & 2 & 6 & 6 & 2 \\
4 & 4 & 24 & 4 & 2 & 6 & 2 & 6 & 2 & 6 \\
4 & 4 & 4 & 24 & 2 & 2 & 6 & 2 & 6 & 6 \\
6 & 6 & 2 & 2 & 4 & 2 & 2 & 2 & 2 & 1 \\
6 & 2 & 6 & 2 & 2 & 4 & 2 & 2 & 1 & 2 \\
6 & 2 & 2 & 6 & 2 & 2 & 4 & 1 & 2 & 2 \\
2 & 6 & 6 & 2 & 2 & 2 & 1 & 4 & 2 & 2 \\
2 & 6 & 2 & 6 & 2 & 1 & 2 & 2 & 4 & 2 \\
2 & 2 & 6 & 6 & 1 & 2 & 2 & 2 & 2 & 4
\end{array}\right]
$$

The matrix $L=\Gamma(q) \times B=6 B$

$$
L=\frac{1}{140}\left[\begin{array}{cccccccccc}
24 & 4 & 4 & 4 & 6 & 6 & 6 & 2 & 2 & 2 \\
4 & 24 & 4 & 4 & 6 & 2 & 2 & 6 & 6 & 2 \\
4 & 4 & 24 & 4 & 2 & 6 & 2 & 6 & 2 & 6 \\
4 & 4 & 4 & 24 & 2 & 2 & 6 & 2 & 6 & 6 \\
6 & 6 & 2 & 2 & 4 & 2 & 2 & 2 & 2 & 1 \\
6 & 2 & 6 & 2 & 2 & 4 & 2 & 2 & 1 & 2 \\
6 & 2 & 2 & 6 & 2 & 2 & 4 & 1 & 2 & 2 \\
2 & 6 & 6 & 2 & 2 & 2 & 1 & 4 & 2 & 2 \\
2 & 6 & 2 & 6 & 2 & 1 & 2 & 2 & 4 & 2 \\
2 & 2 & 6 & 6 & 1 & 2 & 2 & 2 & 2 & 4
\end{array}\right]
$$

For optimality to suffice, LHS of the I-optimality equivalence inequality of each design point should be less or equal to $\operatorname{trace}\left[C_{e}^{-1} L\right]=73.9209$ and $\operatorname{trace}\left[C_{u}^{-1} L\right]=$ 64.9382 for EWSCAD and UWSCAD respectively. The LHS of the equivalence theorem given by $f^{\prime}(t) C_{e}^{-1} L C_{e}^{-1} f(t)$ and $f^{\prime}(t) C_{u}^{-1} L C_{u}^{-1} f(t)$ respectively for each design points were given in tables 1 and 2 respectively.

Table 1. I-optimality Equivalence Theorem for EWSCAD.

\begin{tabular}{lllll}
\hline Design & LHS & & $\operatorname{trace}\left[\boldsymbol{C}_{\boldsymbol{e}}^{-\mathbf{1}} \boldsymbol{L}\right]$ & Optimality \\
\hline$\eta_{1}$ & 316.729 & $>$ & 73.9209 & Not optimal \\
$\eta_{2}$ & 48.904 & $<$ & 73.9209 & Optimal \\
$\eta_{3}$ & 12.565 & $<$ & 73.9209 & Optimal \\
$\eta_{4}$ & 5.360 & $<$ & 73.9209 & Optimal \\
\hline
\end{tabular}

Table 2. I-optimality Equivalence Theorem for UWSCAD.

\begin{tabular}{lllll}
\hline Design & LHS & & $\operatorname{trace}\left[\boldsymbol{C}_{\boldsymbol{u}}^{-\mathbf{1}} \boldsymbol{L}\right]$ & Optimality \\
\hline$\eta_{1}$ & 292.042 & $>$ & 64.9382 & Not optimal \\
$\eta_{2}$ & 36.354 & $<$ & 64.9382 & Optimal \\
$\eta_{3}$ & 9.676 & $<$ & 64.9382 & Optimal \\
$\eta_{4}$ & 3.700 & $<$ & 64.9382 & Optimal \\
\hline
\end{tabular}

The design points $\eta_{2}, \eta_{3} a n d \eta_{4}$, for the two designs attained the optimality according to the equivalence theorem. 
The I-efficiency given by the equation (14) was utilized to compare the efficiencies of the two designs namely the EWSCAD and UWSCAD. It showed that $I_{\text {eff }}=\frac{64.9382}{73.9209} \times$ $100=87.85 \%$, meaning that the EWSCAD was a better design than the UWSCAD.
A concrete experiment produced the regression model as given by (18) as was shown by [9]. $E(\hat{Y})$ is the estimated compressive strength of concrete, while $t_{1}, t_{2}, t_{3}$ and $t_{4}$ represented Water, Cement, Fine aggregate (Sand) and Coarse aggregate (Ballast), respectively.

$$
E(\hat{Y})=23.41 t_{1}^{2}+37.95 t_{2}^{2}+37.31 t_{3}^{2}+28.39 t_{4}^{2}+34.93 t_{1} t_{2}+53.46 t_{1} t_{3}+60.53 t_{1} t_{4}+38.83 t_{2} t_{3}+48.34 t_{2} t_{4}+59.27 t_{3} t_{4}
$$

Table 3 represents the ANOVA table for the model (18).

Table 3. Summarized Analysis of Variance for the concrete experiment.

\begin{tabular}{lllll}
\hline $\begin{array}{l}\text { Sources of } \\
\text { variations }\end{array}$ & $\begin{array}{l}\text { Degrees of } \\
\text { freedom }\end{array}$ & $\begin{array}{l}\text { Sum of } \\
\text { Squares }\end{array}$ & MSS & F \\
\hline Regression & 9 & 136.703 & 15.189 & 2.10 \\
Residual & 35 & 253.102 & 7.231 & \\
Total & 44 & 389.905 & & \\
\hline
\end{tabular}

The $\mathrm{F}$ calculated value is 2.10 and $F_{(0.1,9,35)}=1.79$, this showed that the model estimates were significant meaning that compressive strength of concrete varied on the different combinations of the components.

A stationary point of a response surface may be obtained by use of Canonical analysis, where the regression model is transformed to a new co-ordinate system, but the most straightforward way is to examine a contour plot of the fitted model as indicated by [8]. The data obtained from the experiment for this study was presented using the contours and response surfaces.

The Figure 1 is the data boxplot that gives the descriptive statistics of the concrete experiment. It indicated that the median of the data is approximately $27.5 \mathrm{~N} / \mathrm{mm}^{2}$. It also shows that the reading $31.509 \mathrm{~N} / \mathrm{mm}^{2}$ is an outlier.

\section{Boxplot}

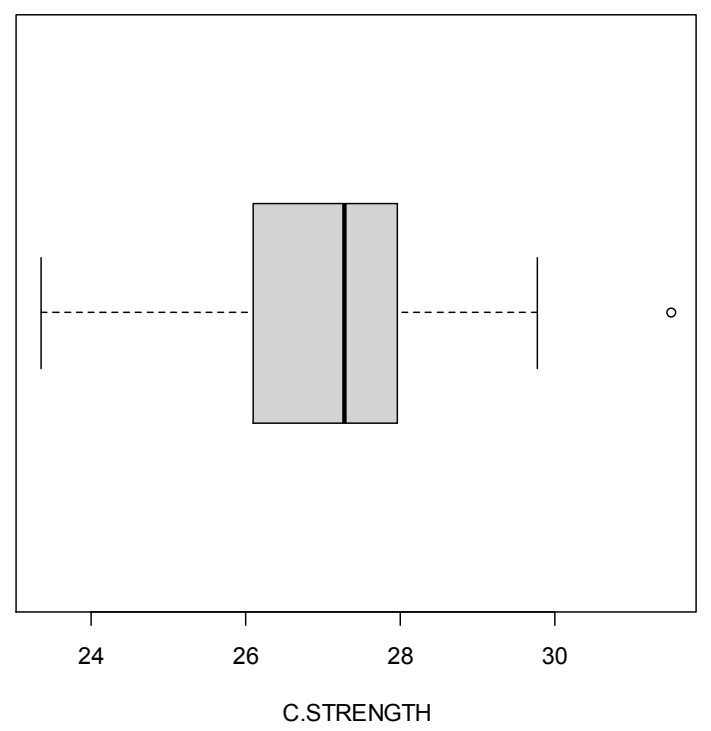

Figure 1. Concrete data Box plot.

Figures 2, 3 and 4 shows the image, contours and the response surface respectively of one of the $4 C_{2}$ outcomes of the experiment. It shows how compressive strength was affected due to water and cement interaction. At a constant ratio of 0.4 of cement there was a steep descent of compressive strength as water was increased.

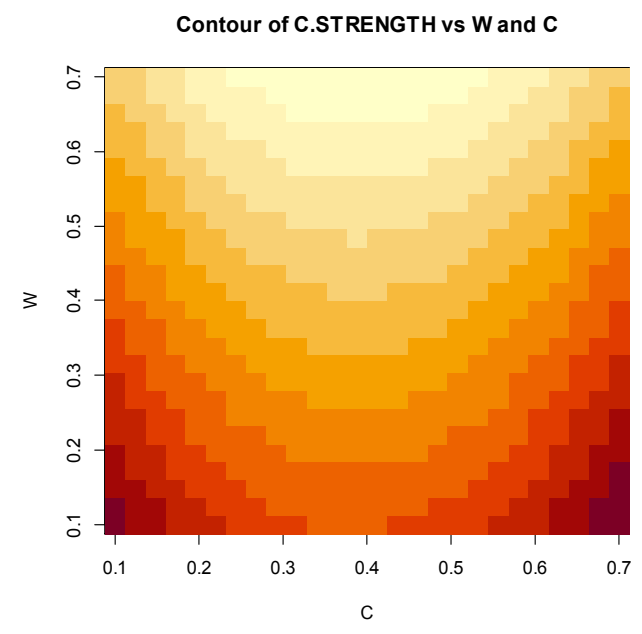

Figure 2. Contour Image for Water vs. Cement.

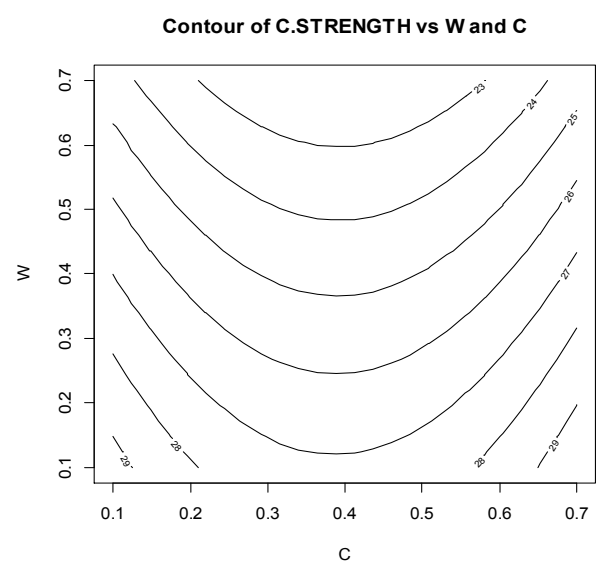

Figure 3. Contours for Water vs. Cement. C.STRENGTH vs W and C

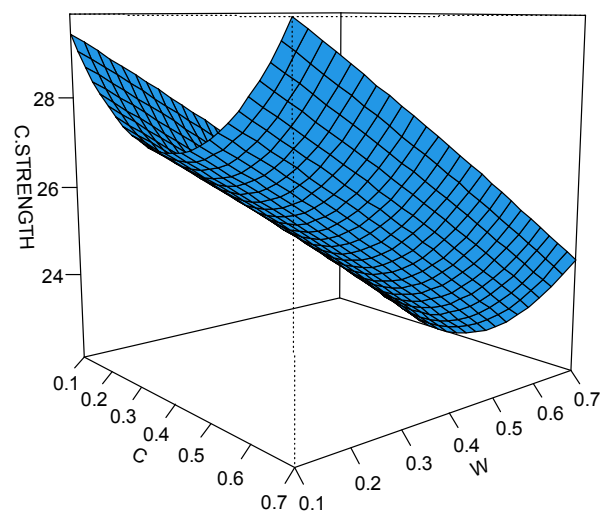

Figure 4. Response surface for Water vs. Cement. 
The $L_{2}$ matrix (19) of the concrete experiment Kronecker model below was obtained by the use of the integrals summarized in (16) and the betas in the regression model (18).

The RHS I-Optimality values of the I-equivalence theorem

$\left[\begin{array}{cccccccccc}15.658 & 4.231 & 4.159 & 3.165 & 5.841 & 8.939 & 10.121 & 2.164 & 2.694 & 3.304 \\ 4.231 & 41.147 & 6.742 & 5.130 & 9.469 & 4.830 & 5.469 & 10.526 & 13.104 & 5.355 \\ 4.159 & 6.742 & 39.772 & 5.044 & 3.103 & 14.427 & 5.377 & 10.348 & 4.294 & 15.795 \\ 3.165 & 5.130 & 5.044 & 23.028 & 2.361 & 3.614 & 12.275 & 2.625 & 9.803 & 12.019 \\ 5.841 & 9.469 & 3.103 & 2.361 & 5.810 & 4.460 & 5.034 & 3.229 & 4.020 & 2.465 \\ 8.939 & 4.830 & 14.427 & 3.614 & 4.460 & 13.609 & 7.705 & 4.943 & 3.076 & 7.544 \\ 10.121 & 5.469 & 5.377 & 12.275 & 5.034 & 7.705 & 17.447 & 2.798 & 6.967 & 8.542 \\ 2.164 & 10.526 & 10.348 & 2.625 & 3.229 & 4.943 & 2.798 & 7.180 & 4.469 & 5.480 \\ 2.694 & 13.104 & 4.294 & 9.803 & 4.020 & 3.076 & 6.967 & 4.469 & 11.127 & 6.822 \\ 3.304 & 5.355 & 15.795 & 12.019 & 2.465 & 7.544 & 8.542 & 5.480 & 6.822 & 16.728\end{array}\right]$

for the Concrete model of EWSCAD were obtained from (20) the matrix $C_{e}^{-1} L_{2}$. Where $C_{e}^{-1}$ is the inverse of matrix (18) as done by [9].

EWSCAD and UWSCAD as shown in [Table 4]. The efficiency of the two designs on the concrete experiment using (14) was, $\quad I c_{\text {eff }}=\frac{13,902.12}{17,477.51} \times 100=79.54 \%$. EWSCAD turned out to more I-efficient than UWSCAD.

\section{Conclusion}

The results on I-optimality and efficiency for the inscribed tetrahedral design and the concrete experiment were identical.

The concrete model has showed that the designs $\eta_{2}, \eta_{3}$ and $\eta_{4}$ achieved the optimality condition, for both

$\left[\begin{array}{cccccccccc}740.35 & 386.99 & 203.52 & 107.95 & 109.90 & 17.642 & -39.45 & 204.12 & 255.92 & 271.83 \\ 710.19 & 4090.38 & 1117.90 & 767.11 & 867.70 & 913.90 & 1012.37 & 944.58 & 1101.53 & 978.84 \\ 289.31 & 634.88 & 3244.16 & 370.73 & 296.22 & 726.81 & 529.08 & 617.71 & 446.76 & 783.75 \\ -39.84 & 52.28 & 24.28 & 1139.65 & 40.88 & 95.63 & -44.06 & 71.95 & 51.17 & -5.35 \\ -1222.6 & -2344.04 & -19.45 & -519.16 & -164.25 & -1107.08 & -1570.7 & -765.23 & -1217.99 & 122.69 \\ 390.29 & 864.82 & -357.02 & -379.26 & 181.22 & 1886.89 & -453.14 & -34.99 & 354.02 & -748.68 \\ 1034.44 & 728.49 & 29.82 & 673.66 & 382.19 & 191.86 & 3236.62 & 312.24 & 207.43 & -115.1 \\ -248.58 & -1989.78 & -2579.99 & -509.43 & -655.16 & -1377.44 & 26.25 & -126.62 & -1101.15 & -1699.13 \\ -147.76 & -510.28 & 153.51 & -692.34 & -273.36 & 291.7 & -928.16 & -251.06 & 1058.09 & -98.72 \\ -609.20 & 106.91 & 113.44 & 250.06 & -98.72 & -615.16 & -723.93 & -106.65 & -170.74 & 2372.25\end{array}\right]$

Likewise, the RHS I-Optimality values of the I-equivalence theorem for the Concrete model of UWSCAD were obtained. The matrix (21) which is $C_{u}^{-1} L_{2}$ was obtained from the product of matrix $L_{2}$ (19) above and inverse of matrix $C_{u}$ (19) of [9].

$\left[\begin{array}{cccccccccc}732.18 & 442.61 & 248.64 & 95.58 & 123.99 & 41.82 & -30.63 & 188.26 & 213.11 & 206.87 \\ 615.27 & 3889.48 & 1047.52 & 672.30 & 797.96 & 771.85 & 827.48 & 885.26 & 1015.96 & 825.01 \\ 256.46 & 273.64 & 3121.01 & 334.24 & 260.72 & 687.81 & 415.39 & 605.64 & 388.32 & 742.33 \\ -31.62 & 164.91 & 103.14 & 1132.30 & 37.46 & 65.67 & -17.34 & 79.45 & 95.60 & 40.54 \\ -889.19 & -2021.14 & -106.53 & -384.93 & -183.35 & -731.27 & -1013.83 & -589.28 & -887.73 & -101.41 \\ 183.73 & 516.68 & -597.91 & -202.07 & 120.21 & 1182.02 & -222.59 & -70.35 & 154.52 & -497.72 \\ 645.51 & 569.25 & 185.11 & 370.07 & 299.41 & 244.59 & 2186.44 & 217.73 & 206.83 & 52.60 \\ -276.86 & -1852.20 & -2412.19 & -431.15 & -545.03 & -1032.21 & -233.69 & -249.23 & -835.69 & -1230.35 \\ -141.08 & -830.14 & 48.84 & -600.51 & -257.32 & 48.87 & -611.16 & -244.15 & 582.12 & -508.03 \\ -312.16 & 152.68 & -264.04 & 63.85 & -56.26 & -378.34 & -367.92 & -76.73 & -56.13 & 1509.16\end{array}\right]$

The design points $\eta_{2}, \eta_{3}$ and $\eta_{4}$, for the two designs and the experiment attained the same optimality conditions by use of the I-optimal equivalence inequality. EWSCAD was a more efficient design than UWSCAD. The response surface shown by figure 2 showed that the lowest line of descent also fell on the I-optimal design points. The I-optimality criterion being one that reduces average prediction variance, does optimize outcomes more than any other criteria does. For further studies, another simplex centroid axial design may be used to evaluate the same I-optimality conditions to create an experimental design, which would otherwise be evaluated for the same.

Table 4. Optimality values for the Concrete model.

\begin{tabular}{lllll}
\hline & Design point & $\boldsymbol{L} \boldsymbol{H} \boldsymbol{S}_{\boldsymbol{e}}$ & $\boldsymbol{L} \boldsymbol{H} \boldsymbol{S}_{\boldsymbol{u}}$ & Remark \\
\hline \multirow{4}{*}{$\eta_{1}$} & $0.1[7,1,1,1]$ & $19,021.4$ & $17,196.14$ & Not Optimal \\
& $0.1[1,7,1,1]$ & $77,562.46$ & 69.009 .86 & Not Optimal \\
& $0.1[1,1,7,1]$ & $59,897.66$ & $54,036.29$ & Not Optimal \\
& $0.1[1,1,1,7]$ & $26,686.15$ & $24,253.16$ & Not Optimal \\
& $0.1[4,4,1,1]$ & $8,070.3$ & $4,991.74$ & Optimal \\
$\eta_{2}$ & $0.1[4,1,4,1]$ & $12,563.3$ & $7,034.65$ & Optimal \\
& $0.1[4,1,1,4]$ & $12,509.7$ & $6,246.03$ & Optimal \\
& $0.1[1,4,1,4]$ & $12,103.6$ & $7,214.64$ & Optimal \\
\hline
\end{tabular}




\begin{tabular}{lllll}
\hline & Design point & $\boldsymbol{L H S}_{\boldsymbol{e}}$ & $\boldsymbol{L} \boldsymbol{H} \boldsymbol{S}_{\boldsymbol{u}}$ & Remark \\
\hline & $0.1[1,1,4,4]$ & $14,231.8$ & $7,954.72$ & Optimal \\
& $0.1[1,4,4,1]$ & $10,250.9$ & $6,803.42$ & Optimal \\
& $0.1[3,3,3,1]$ & $1,922.27$ & $1,397.19$ & Optimal \\
$\eta_{3}$ & $0.1[3,3,1,3]$ & $2,140.67$ & $1,513.79$ & Optimal \\
& $0.1[3,1,3,3]$ & $3,085.06$ & $2,076.86$ & Optimal \\
& $0.1[1,3,3,1]$ & $2,426.19$ & $1,738.49$ & \\
$\eta_{4}$ & {$\left[\frac{1}{4}, \frac{1}{4}, \frac{1}{4}, \frac{1}{4}\right]$} & 819.462 & 595.557 & Optimal \\
\hline
\end{tabular}

\section{References}

[1] Atkinson A. C., Donev A. N., Tobias R. D (2007). Optimum Experimental Designs, with SAS. Oxford University Press, Oxford.

[2] Cornell, J. A. (2002). Experiments with Mixtures, Third Edition. John Wiley \& Sons Inc, New York.

[3] Draper N. and John R. (1977). Designs in three and four components for mixture models with inverse terms. Technometrices, 19, 17-30.

[4] Draper, N. R. and Pukelsheim, F. (1998). Mixture models based on homogeneous polynomials. Journal of statistical planning and inference, $71, \mathrm{pp} .303-311$.

[5] Fedorov, V. (1972). Theory of optimal experiments. Academic Press, New York.

[6] Jones B., and Goos P., (2012). I-optimal Versus D-optimal Slit-plot response surface designs. Journal of Quality Technology vol 44 (2).

[7] Kerich G., Koske J., Rutto M., Korir B., Ronoh B., Kinyanjui J.,
Kungu P., (2014). D-Optimal Designs for Third-Kronecker Model Mixture Experiments with an Application to Artificial Sweetener Experiment. IOSR Journal of Mathematics vol 10 (6). $p p$ 32-41.

[8] Montgomery D. C., (2001). Design and Analysis of Experiments $5^{\text {th }}$ Ed. John Wiley and Sons, Inc.

[9] Njoroge E. W., Koske, J., Mutiso J. (2020). D- and G- Optimal Axial Slope Designs for Four Ingredient Mixture. Journal of Applied Mathematics and Physics vol 8 (1). pp 20-25.

[10] Njoroge E. W., Koske, J., Mutiso J. (2020). Optimization of Plinth Concrete Mix Using Quad-Axial Weighted Simplex Centroid Designs And Second Degree Kronecker Model. Not published.

[11] Peter G., Bradely J. and Utami S. (2013) I- optimal mixture designs.www.repec.org, Research Papers in Economics D/2013/1169/033.

[12] Rady, E. A., Abd EL-Monsef, M. M. E., Seyam, M. M., (2009). Relationship among several optimality criteria. Interstat Journal volume 15 (6). Pp 1-11.

[13] Sinha B. K., Das P., Mandal N. K. and Pal M., (2010). Parameter Estimation of Linear and Quadratic Mixture Models. A review. Pak. Statist, vol 26 (1), 77-96.

[14] Sinha B. K., Das P., Mandal N. K. and Pal M., (2014). Optimal Mixture Experiments. http://www.Springer.com/978-81-322-1785-5.

[15] Ugbe T. A. and Akpan S. S., (2013). On the comparision of the boundary and interior support points of a response surface under optimality criteria. International journal of Mathematics and Statistics studies. Vol 1 (4), pp 48-58. 